\title{
More than machines
}

\author{
As artificial intelligence, robotics and machine learning are high on the agenda everywhere, Nature Machine \\ Intelligence launches to stimulate collaborations between different disciplines.
}

U: nderstanding human thought has for centuries kept the brightest minds busy, with many of the key questions remaining unanswered. This hasn't stopped us from imagining machines that imitate our thoughts and behaviour. Such visions of intelligent machines have influenced how we contemplate our humanity and have given us popular narratives such as those pondering the coexistence of androids and humans, or dystopian tales where a super-intelligent power has taken over the world. More pervasively, the pursuit of machines that mimic some of our intelligence and carry out useful tasks has played a key role in shaping the technology that underpins societal transformations, from the industrial revolution to the digital age.

The development of intelligent machines seems to be gathering pace, as we regularly hear about an artificial intelligence (AI) revolution. However, contrary to reports, we are not witnessing some sort of technological singularity: the eye-catching advances from the past few years are largely a continuation from decades of sustained research and funding. For instance, selfdriving cars autonomously sharing the road with other traffic were already demonstrated in a test in 1994 in Paris ${ }^{1}$. That machine learning would be of great industrial use was also well understood in the early $1990 \mathrm{~s}^{2}$. A telerobotic spacecraft landed on Mars and sent back pictures in 1997.

This is not to say that there hasn't been a shift in impact. There are several factors underlying this shift, such as the advance in computer processing power, but perhaps most importantly the availability of huge amounts of data since the late 1990s.

These developments were in turn due to the spread of affordable mobile devices that collect data on a massive scale, the ability to share data as well as the accelerating storage capacity. As a result, deep learning, which can recognize patterns in complex datasets when there is sufficient data to train the system on, made its famous breakthroughs around 2012. Deep learning is considered as a form of $\mathrm{AI}$, as it bears similarities to human-based learning. Indeed, the past few years have seen remarkable demonstrations of deep learning performing tasks that humans are typically skilled at: playing and winning - strategic games, medical diagnosis at the level of human experts ${ }^{3}$, speech recognition and language translation.

On the one hand this form of AI is considered 'narrow', because deep learning systems are typically trained for one specific task. However, scientists from a wide range of fields are becoming aware that the impact of deep learning is far from 'narrow': a well-thought-out application could be transformative for a field, tackling fundamental questions and enabling fast progress - for example, in finding new materials, identifying important signals in particle physics or astrophysical data, discovering links between genetics and phenotypes, and in classifying cellular tissues in microscopic images.

With every advanced technology that becomes available, the risk of misuse and unwanted societal implications needs to be examined. These ethical issues are all the more relevant for AI given that it is a decentralized phenomenon, in which substantial power has been handed to those companies and institutes with natural access to much data. Ethical concerns around the development and use of $\mathrm{AI}$ are taken increasingly seriously. Such concerns have led to widespread efforts to develop laws, principles and standards to promote the human-friendly use of AI and to start research programmes studying its societal implications ${ }^{4-6}$.

There is no doubt that the deeply ingrained desire to understand and mimic intelligence will continue to inspire technological and scientific innovations. Nature Machine Intelligence will endeavour to bring different fields together, forging new collaborations in AI, robotics, cognitive science and machine learning, to further develop visions of intelligent machines that can be of inspiration and use for humanity. There are roughly three main themes that we will initially focus on: the engineering and study of algorithms and hardware to build intelligent machines; applications of machine intelligence (such as deep learning systems) to specific areas and topics in other domains (for example, physics, biology and healthcare); and lastly the study of the impact of machine intelligence in society, industry and science.

This first issue of Nature Machine Intelligence features pieces in each of these categories. In an Article, Etienne Burdet and colleagues report on work of the first category, on developing algorithms for adaptive human-robot collaboration, and the Review by Kenneth Stanley et al. presents an overview of approaches that combine deep learning with evolutionary algorithms. For the second theme, one of the best-known applications of deep learning is in medicine, and a Perspective by Edmon Begoli and co-workers examines the need for uncertainty quantification in this area. In a Comment, Stephen Cave and Seán ÓhÉigeartaigh discuss the different timescales of ethical concerns in AI, falling in the third category.

We also start a series called Challenge Accepted, which are reports on data challenges and competitions in AI and robotics, to highlight the important and sometimes surprising role these play in steering a field, giving young researchers a chance to demonstrate their skills and to crowdsource solutions to outstanding practical questions in science or industry.

The pursuit of intelligent machines will continue to inspire in many ways, providing us with insights into human intelligence as well as stimulating technological and scientific innovation that could lead to future societal transformations. Now is the time to be part of the conversation.

Published online: 7 January 2019 https://doi.org/10.1038/s42256-018-0014-z

References

1. Delcker, J. Politico https://go.nature.com/2QOfrxP (24 June 2018).

2. Jordan, M. Medium https://go.nature.com/2Bedwss

(19 April 2018).

3. De Fauw, J. et al. Nat. Med. 24, 1342-1350 (2018).

4. AI Now; https://ainowinstitute.org

5. Human-Centred AI; http://hai.stanford.edu

6. AI4People; http://www.eismd.eu/ai4people 